\title{
THE ASYMPTOTIC NUMBER OF CONVEX POLYHEDRA
}

BY

\author{
L. B. RICHMOND AND N. C. WORMALD
}

\begin{abstract}
We obtain an asymptotic formula for the number of combinatorially distinct convex polyhedra with $n$ edges.
\end{abstract}

1. Introduction. The number of combinatorial polyhedra has been studied by many authors, perhaps first of all by Euler. A very interesting account up to 1975 has been given by P. J. Frederico [2]. By a well-known theorem of Steinitz a convex polyhedron is combinatorially equivalent to a 3-connected planar map (with more than three edges). B. Grünbaum's proof [3] is the most elegant known to these authors. Indeed Mani [5] has shown that for each 3-connected planar map $M$ there is a convex polyhedron whose 1-skeleton is isomorphic to $M$ and such that every automorphism of $M$ is induced by an isometry of the convex polyhedron. The enumeration of planar maps has progressed considerably following the breakthrough of W. T. Tutte in the early sixties. Tutte [6] has given a survey of the methods and results known up to 1973 . Until quite recently most of the progress concerned the enumeration of rooted planar maps. A planar map is said to be rooted when one directed edge in it is distinguished as the root and when two sides of the root are distinguished as "left" and "right". In fact Tutte [7] has shown that the number $R(n)$ of rooted 3-connected planar maps with $n$ edges is asymptotic to

$$
\frac{2 n^{-5 / 2} 4^{n}}{243 \sqrt{\pi}}
$$

The purpose of this paper is to obtain an asymptotic estimate for $M(n)$ the number of 3-connected planar maps with $n$ edges. In fact Theorem 2.8 states that

$$
4 n M(n)=R(n)+O(\exp (1.384 n)) .
$$

Since $2 \log 2=1.38629436 \mathrm{i} 1 \ldots$, and in view of Steinitz's result this gives an asymptotic formula for the number of convex polyhedra with $n$ edges. We have not relied on Mani's result and used geometric arguments, as a purely combinatorial approach may succeed for maps which are not 3-connected. Our approach is similar to that of Tutte [8] who has obtained an asymptotic formula for the number of convex polyhedra with $2 n$ faces, all triangular. We depend very essentially on W. G. Brown's results [1] concerning rooted nonseparable planar maps.

Received by the editors August 3, 1981.

1980 Mathematics Subject Classification. Primary 05C30, 05C10.

'Research supported by National Science Foundation Grant MCS-8101555.

Research supported by NSERC grant A4067. 
We should make the following remarks concerning exact enumeration. Recently N. C. Wormald [9] has given a formula for the number of unrooted maps with $n$ edges and plans to count the number of 3-connected planar maps. Also V. A. Liskovets [4] has obtained very elegant results for the enumeration of maps up to orientation-preserving homeomorphisms, which imply that almost all 3-connected planar maps have no nontrivial orientation preserving automorphisms. Since these automorphisms are the easiest ones to handle by our method, we do not rely on Liskovets' result. We are grateful to Tutte for several discussions of this problem.

2. 3-connected maps and nonseparable maps. Lemmas A.1, A.2 and A.3 are stated and proved in the Appendix. Suppose $M$ is a 3-connected map with $n$ edges and with a nontrivial automorphism $\alpha$ of minimal order which will be a prime. As usual we regard $M$ as a dissection of a sphere $\Pi$ into the vertices, edges and faces of $M$ (called the cells of $M$ ). We say $\alpha$ is sense-preserving if it preserves the orientation of $\Pi$, and sense-reversing otherwise. (This concept can alternately be defined combinatorially by studying the action of $\alpha$ on the cells of $M$.) An invariant of $\alpha$ is a cell of $M$ fixed by $\alpha$.

If $\alpha$ is sense-reversing then it has even order and hence order 2. So altogether, $\alpha$ must fall into one of the following three classes. Firstly, we say $\alpha$ is plane-reflective if it is sense-reversing of order 2 and has at least one invariant. (By Mani's result this would imply that $\alpha$ corresponds to the reflection of some convex polyhedron in a plane.) This seems to be the most troublesome type of automorphism to deal with in terms of the object of this paper. Secondly, we say $\alpha$ is antipodal if it is sense-reversing and has no invariant (corresponding to a reflection of a polyhedron in some point). Thirdly, we say $\alpha$ is rotative if it is sense-preserving. (This corresponds to a rotation of a polyhedron.)

Since we prefer to argue independently of Mani's result we need to establish a few facts about the action of $\alpha$ on $M$. The following three lemmas do not depend upon the 3-connectedness of $M$. The first two are proved here by the same type of argument which Tutte [8] applied to 3-connected maps in which every face is a triangle. We first define a cell-cycle of $M$ to be any set $S$ of cells such that the graph $G$, whose vertex set is $S$ and where two vertices of $G$ are adjacent in $G$ if and only if they are incident cells in $M$, is a cycle. A cell-path is defined in the obvious analogous way.

LEMMA 2.1. If $\alpha$ is plane-reflective then the set of invariants of $\alpha$ is a cell-cycle.

Proof. The cyclic order of cells incident with any given invariant $c$ of $\alpha$ is reversed by $\alpha$, and it follows that $c$ is incident with precisely two other invariants. This is also true for any other invariant. Since $M$ is finite, there is consequently a set $S$ of invariants, $c \in S$, such that each cell in $S$ is incident with just two others in $S$, and such that there is a simple closed curve contained in the union of cells in $S$ and passing through each of them. Removing this curve from $\Pi$ leaves 2 simply-connected domains by the Jordan Curve Theorem. All the noninvariant cells contained in one of these domains are mapped by $\alpha$ to cells in the other. (This is certainly true of the cells incident with any cell in $S$, because $\alpha$ is sense-reversing, and all the rest 
follow by simple-connectedness.) Thus there are no invariants not in $S$ and the proof is complete.

LEMMA 2.2. If $\alpha$ is antipodal then there is a cell-cycle of $M$ which is fixed setwise by $\alpha$ and contains no vertices.

Proof. Let $F$ be a face of $M$. Since $\alpha$ is antipodal, $\alpha(M) \neq M$. Let $G$ be the graph whose vertices are the faces of $M$, with adjacency in $G$ determined by adjacency in $M$ (so that $G$ is the defining graph of the dual map of $M$ ). Let $P(F)$ be a shortest path in $G$ joining $F$ to $\alpha(F)$, and suppose that $F$ is chosen so that $P(F)$ has the shortest length possible. Let $S_{1}$ be the set of faces of $M$ which are internal vertices of $P(F)$, and let $S_{2}$ be the set of images of faces in $S_{1}$ under $\alpha$. We next show that $S_{1} \cap S_{2}$ is empty. For if $F^{\prime}$, say, is in $S_{1}$ and $S_{2}$, then as $\alpha$ is of order 2, $\alpha\left(F^{\prime}\right) \in S_{1} \cap S_{2}$ also. So the cells of $S_{1}$ provide a path in $G$ from $F^{\prime}$ to $\alpha\left(F^{\prime}\right)$, which contradicts the minimality of the length of $P$. It follows now that the faces in $S_{1} \cup S_{2} \cup\{F, \alpha(F)\}$, together with the edges of $M$ which separate faces of $M$ which are adjacent in $P(F)$, together with the images of these under $F$, form a cell-cycle of the required type.

The next lemma has been used several times in the enumeration of unrooted maps. It is proved, but certainly not for the first time, in Wormald [10] using a result from topology. It can also be proved fairly easily using an entirely combinatorial argument of the type found in Tutte $[8, \S 5]$.

LEMMA 2.3. If $\alpha$ is rotative then it has precisely two invariants.

This lemma will be used later on to obtain a cell-cycle which is suitable for our scheme when $\alpha$ is rotative.

Basically, these cell-cycles which we have been proving to exist in $M$ will be used to find a piece of $M$ which determines the whole of $M$. Hopefully the number of possibilities for that piece is much smaller than the number of rooted 3-connected planar maps. To establish this we try to use the piece of $M$ to construct a fairly well-connected map $N$ with not too many edges. The number of possibilities for $N$ will bear some relationship to the number of possibilities for that piece of $M$ and consequently also to the number of possibilities for $M$. The next definitions and two lemmas are concerned with finding a suitable map $N$.

First let us say that an edge is flipped by $\alpha$ if it is fixed by $\alpha$ but its incident vertices are not. All other invariant edges are transfixed by $\alpha$. If $\alpha$ is plane-reflective then by Lemma 2.1 the set of invariants of $\alpha$ is a cell-cycle, and this cell-cycle clearly does not consist of three cells mutually incident. Hence the boundary of the union of the invariants of $\alpha$ consists of two simple closed curves which may intersect but do not cross each other, in view of the fact that each invariant is incident with only two others. Let $R_{1}$ and $R_{2}$ be the closures of the simply-connected regions of $\Pi$ cut off by these curves which do not contain invariants of $\alpha . R_{1}$ and $R_{2}$ are interchanged by $\alpha$. Define $M_{1}$ to be the map whose edges and vertices are just the edges and vertices of $M$ within $R_{1}$, which automatically include the edges of $M$ transfixed by $\alpha$ and the vertices fixed by $\alpha$. Also, take a simple closed curve $C$ contained in the union of the 
invariants of $\alpha$ and intersecting each of them, in particular, intersecting each flipped edge at a unique point and define a map $M_{2}(C)$ as follows: the vertices of $M_{2}(C)$ are the vertices of $M$ in $R_{1}$ together with the points of intersection of $C$ with the flipped edges of $M$, and the edges of $M_{2}(C)$ are the edges of $M$ in $R_{1}$ (thus including each transfixed edge) together with each interval of $C$ contained in a (fixed) face of $M$, and also each of the portions of the flipped edges between the new vertices on $C$ and the vertices of $M$ on the boundary of $R_{1}$.

The reader may find it useful to think of the polyhedron which realizes $M$ having a plane of reflection intersecting its surface in the curve $C . M$ is the part which lies completely in or completely on one side of the plane. $M_{2}$ corresponds to the polyhedron formed by $M$ and the plane of reflection, i.e. having a piece of the plane as a face. Of course this interpretation uses Mani's result as well as the Steinitz theorem but suggests the following lemma:

LEMMA 2.4. If $\alpha$ is plane-reflective then $M_{1}$ is nonseparable and $M_{2}(C)$ is 3-connected.

Proof. Observe that $R_{1}$ is simply-connected, from its definition, as the region cut off by a simple closed curve, and that its boundary, which it contains, consists entirely of edges and vertices of $M$ and hence $M_{1}$. Thus each pair of vertices of $M$ on the boundary of $R_{1}$ are 2-connected to each other (i.e. connected by 2-internally-disjoint paths) in the defining graph, $G$, say, of $M_{1}$. Also since $M$ is 3-connected and the boundary of $R_{1}$ is a simple curve it follows from easy connectivity arguments that there are at least 2 internally-disjoint paths in $G_{1}$ connecting any vertex in the interior of $R_{1}$ to 3 distinct points in the boundary of $R_{1}$. It follows that $G_{1}$, and hence $M_{1}$, is nonseparable.

Let $G_{2}$ be the defining graph of $M_{2}$, and observe that $G_{1} \subseteq G_{2}$. Then by the preceding remarks, each vertex interior to $R_{1}$ is connected in $G_{2}$ to at least 3 distinct vertices on the boundary of $R_{1}$ by pairwise internally disjoint paths. Also, any vertex $v$ of $G_{2}$ is not in $G_{1}$ corresponds to an interior point of an edge $E$ of $M$ flipped by $\alpha$, and $v$ is connected to the end vertex of $E$ in $R_{1}$ as well as lying in the cycle of vertices and eges of $M_{2}(c)$ whose union is $C$. Since $M$ is 3-connected, the number of flipped edges plus the number of vertices lying in $C \cap R_{1}$ is at least 3 and hence $v$ is connected in $G_{2}$ by pairwise internally disjoint paths to 3 distinct vertices in the boundary of $R_{1}$. Thus, to prove that $G_{2}$ is 3-connected it is enough to show that the vertices on the boundary of $R_{1}$ are 3-connected to each other in $G_{2}$. Assume this is false-then since the boundary of $R_{1}$ determines a cycle in $G_{2}$, there must be two vertices $v_{1}$ and $v_{2}$ in $T$ whose removal from $M_{2}$ separates at least two other vertices in $T$ from each other. Clearly $v_{1}$ and $v_{2}$ are not consecutive in $T$, and both are incident in $M$ with the same face $F$ inside $R_{1}$. So if $v_{1}$ and $v_{2}$ both lie on $C$, i.e. are invariants of $\alpha$, then the face $\alpha(F)$ which lies in $R_{2}$ is incident in $M$ with $v_{1}$ and $v_{2}$. But two vertices of the 3-connected map $M$ cannot both be incident with the same two faces. Hence at least one of $v_{1}$ and $v_{2}$, say $v_{1}$, does not lie in $C$.

Suppose that $v_{1}$ is not an end of an edge flipped by $\alpha$. Then $v_{1}$ is incident with just one face of $M_{2}$ outside $R_{1}$; call the face $F_{1} . F_{1}$ cannot be incident with $v_{2}$ for it is a 
subset of a face of $M$ and again $v_{1}$ and $v_{2}$ cannot both be incident with the same 2 faces of $M$. Thus the boundary of $F_{1}$ provides a path in $M_{2}$ which avoids $v_{1}$ and $v_{2}$ but joins the two points $u_{1}$ and $u_{2}$ on either side of $v_{1}$ in the cycle $T_{1}$ which contradicts our choice of $v_{1}$ and $v_{2}$. So $v_{1}$ must be the end of an edge flipped by $\alpha$. But then it is incident with two faces outside $R_{1}$ and for the same reasons as before neither of these faces is incident with $v_{2}$ and their two boundaries again provide a $\left(u_{1}, u_{2}\right)$-path in $M_{2}$ avoiding $v_{1}$ and $v_{2}$. This is again a contradiction and the proof of the lemma is complete.

We shall deal with antipodal and rotative automorphisms in a slightly different way, using the next lemma.

Let $C$ be any simple closed curve in $\Pi$ passing through no vertices of $M$, intersecting no edge in more than one point, and intersecting at least 2 faces of $M$ (hence at least 3 faces since $M$ is 3-connected) and no more than two edges incident with any one face. Let $R_{1}$ and $R_{2}$ be the two simply-connected open regions of $\Pi$ which have $C$ as their boundaries. Let $N_{1}(C)$ be the map whose vertices and edges are just those vertices and edges of $M$ contained entirely in $R_{1}$. Let $N_{2}(C)$ be a map obtained from $N_{2}(C)$ by adding a new vertex $v$ which lies in $R_{2}$, together with new edges joining $v$ to each of the vertices of $N_{1}(C)$ which is incident with an edge of $M$ intersecting $C$. Let $n_{3}(C)$ be the map whose vertices and edges are the vertices and edges of $N_{1}(C)$, together with new vertices at every point of $C$ which intersects and edge of $M$, and new edges consisting of each protion of $C$ between two such new vertices as well as each portion of an edge of $M$ lying between one of the new vertices and one of $N_{1}(C)$, so that $R_{2}$ is a face of $N_{3}(C)$.

LEMMA 2.5. $N_{2}(C)$ is nonseparable and $N_{3}(C)$ is 3-connected.

Proof. Let $G_{i}$ denote the defining graph of $N_{i}(C)$. Since $C$ is a simple closed curve, it is clear that

(i) the vertices of $N_{1}(C)$ which are incident with the faces of $M$ intersected by $C$ induce a connected subgraph, say $H$, of $G_{1}$.

Since $N_{2}(C)$ is formed from $N_{1}(C)$ by adding a vertex $v$ adjacent only to vertices in $H$, it follows that

(ii) $v$ is not a cut-vertex of $G_{2}$. Let $u$ be any vertex of $M$ inside $R_{1}$. Since $M$ is 3-connected, there are at least three pairwise internally disjoint paths in the defining graph $M$ from $u$ to any given vertex in $R_{2}$. Each of these paths must include an edge intersected by $C$, so they imply that

(iii) each vertex $u$ in $R_{1}$ is connected by at least three pairwise internally-disjoint paths in $G_{2}$ to $v$ and

(iv) each vertex $u$ in $R_{1}$ is connected by at least three pairwise internally-disjoint paths in $G_{3}$ to three distinct vertices of $N_{3}(C)$ lying on $C$.

From (ii) and (iii) we deduce immediately that $G_{2}$ and hence also $N_{2}(C)$ is nonseparable. Since each vertex of $N_{3}(C)$ lying on $C$ is adjacent to a vertex of $H$, we deduce by (i) that any two such vertices are connected in $G_{3}$ by a path all of thse internal vertices are in $H$. Since the edges and vertices of $N_{3}(C)$ contained in $C$ form a cycle in $G_{3}$ it follows that each pair of vertices of $G_{3}$ which lie in $C$ are 3-connected 
to each other. Together with (iv) this implies that $G_{3}$ and hence also $N_{3}(C)$ is 3-connected.

We are now ready to begin some counting. According to Brown [1] if $B_{n, m}$ denotes the number of rooted nonseparable maps with $n$ edges and such that the face to the left of the root-edge is incident with exactly $m$ edges, then we have

$B_{n, m}=\frac{m}{(2 n-m) !} \sum_{j=m}^{\min \{n, 2 m\}} \frac{(3 m-2 j+1)(2 j-m)(j-2) !(3 n-j-m-1) !}{(j-m+1)(n-j) ![(j-m) !]^{2}(2 m-j) !}$.

We use this to obtain a convenient bound on the number of such maps with $m$ large as follows. Observe first that $m \leqslant n$.

LEMMA 2.6. There is a constant $\kappa$ such that if $0.45 \leqslant c \leqslant 1$ and $m=c n$ then

$$
\log B_{n, m}<\left(X_{1} c^{2}+X_{2} c+X_{3}\right) n+\kappa
$$

where $X_{1}, X_{2}$ and $X_{3}$ are defined as in Lemma A.2.

Proof. Let

$$
f(j, m, n)=\frac{(j-2) !(3 n-j-m-1) !}{(n-j) ![(j-m) !]^{2}(2 m-j) !(2 n-m) !}
$$

where $m<j<n$ and $j<2 m$, and let $x$ denote $j / n$. Choose $x$ in the range $c<x<1$ so that $f(j, m, n)$ is maximized. From Stirling's formula for the factorial function, $f(j, m, n)$ is $O\left\{n^{D} \exp \{n G(x, c)\}\right\}$, where $G(x, c)$ is as defined just before Lemma A.2 and where $D$ is an absolute constant. It follows from (2.1) that $B_{n, m}$ is also $O\{n E \exp (n G(x, c))\}$, where $E$ is constant as the cases $j=m, j=n$ and $j=2 m$ clearly cause no problem. The lemma now follows from Lemma A.2 (since the strict inequality in Lemma A.2 swallows up $n^{E}$ ).

We still need to know a little more about the relationship between $M_{1}$ and $M_{2}$ in case $\alpha$ is plane-reflective. It turns out that this relationship is the cause of most of the difficulty of this whole problem, so the following result is important.

LEMMA 2.7. If $\alpha$ is plane-reflective, then the number of flipped edges is at most $n / 3$ and the number of transfixed edges is at most $n / 3$.

Proof. Since $M$ is 3-connected each vertex is incident with at least 3 edges. No vertex of $M$ can be incident with 2 different flipped edges, for that would imply that those two edges had the same pair of ends, contradicting the 3-connectedness of $M$. Thus the number of flipped edges is at most half of the number of vertices of $M$, which is in turn at most $2 n / 3$, so we have the first part of the lemma.

Let $e$ be an edge transfixed by $\alpha$ and let $v$ be a vertex incident with $e$, so that $v$ is invariant of $\alpha$. Since $v$ has degree at least 3 it must be incident with at least two edges which are not invariants of $\alpha$. (Clearly just one such edge is impossible.) Since this is true of every transfixed edge, each such edge is adjacent to at least for noninvariant edges. Conversely, each noninvariant edge $e$ is incident with at most two transfixed edges, for not both vertices incident with $e$ can be invariant, and any 
invariant vertex is incident with at most two transfixed edges by Lemma 2.1. Hence the number of transfixed edges is at most half of the number of noninvariant edges and consequently at most one third of the total number of edges, as required.

The bounds given in (1.1) and Lemma 2.6 on the number of maps can be related easily to the nonseparable and 3-connected maps found in Lemmas 2.4 and 2.5. We next put all these bounds together to deduce our main result. The constant .998 can certainly be lessened slightly by improving our arguments but the best possible value seems to be difficult to pin down.

THEOREM 2.8. The number $M(n)$ of combinatorially distinct 3-connected planar maps on $n$ edges is asymptotic to the number $R(n)$ of rooted 3-connected planar maps on $n$ edges divided by $4 n$. Indeed

$$
4 n M(n)=R(n)\left[1+o(0.998)^{n}\right] .
$$

Proof. Throughout this proof $\kappa$ denotes an absolute constant, perhaps different at each appearance. Inequalities may only hold for $n$ sufficiently large. Let $M$ be a 3-connected map with a nontrivial automorphism of minimal and hence prime order, and with $n \geqslant 4$ edges.

We first treat the case that $\alpha$ is plane-reflective. By Lemma $2.4, M_{1}$ is nonseparable. Let us apply a rooting to $M_{1}$ in such a way that $F$, the face to the left of the root edge is the face $\Pi-R_{1}$, and denote the valency of (the number of edges incident with) $F$ by $l$. Put $t=l / n$. Suppose that $t \geqslant 0.3$. (This is the hardest part of the whole proof.) Let $\alpha$ denote the number of edges transfixed by $\alpha$ and let $b$ denote the number flipped by $\alpha$. Then $M_{1}$ contains all $a$ transfixed edges, no flipped edges, and half of the remaining $n-a-b$ edges. Thus $M_{1}$ has $(n+a-b) / 2$ edges in all. Put $r=(a-b) / l$. Then $r t=(a-b) / n$ so $|r t| \leqslant 1 / 3$ by Lemma 2.7. The number of combinatorially distinct possibilities for $M_{1}$ is at most $B_{p, l}$, where $p=n(1+r t) / 2$, when $M_{1}$ is rooted, so if we ignore the rooting except for specifying which face is $F$, the number of possibilities is still at most $B_{p, l}$. Given such a map, we can reconstruct the combinatorial type of the $M$ if we know $a$ and $b$ and precisely which $a$ edges incident with $F$ are transfixed by $\alpha$ and which $b$ vertices are incident with edges flipped by $\alpha$, and which of the remaining vertices are fixed by $\alpha$. The number of choices for the transfixed edges is at most $\left(\begin{array}{l}l \\ a\end{array}\right)$. Once these are chosen, the vertices incident with them are fixed and there are at least $a$ such vertices, so the number of choices for the $b$ vertices is at most $\left(\begin{array}{c}l-2 \\ b\end{array}\right)$. The remaining vertices fixed by $\alpha$ can be chosen at most $2^{l-a-b}$ ways. Thus the number of possibilities for $M$, given $l, a$ and $b$ is at most

$$
B_{p, l}\left(\begin{array}{l}
l \\
a
\end{array}\right)\left(\begin{array}{c}
l-a \\
b
\end{array}\right) 2^{l-a-b}=B_{p, l} R(l, a, a-b)
$$

where

$$
R(l, a, d)=\left(\begin{array}{l}
l \\
a
\end{array}\right)\left(\begin{array}{l}
l-a \\
a-d
\end{array}\right) 2^{l-2 a+d}=\frac{l ! 2^{l-2 a+d}}{a !(a-d) !(l-2 a+d) !}
$$


Observe that

$$
R(l, a+1, d) / R(l, a, d)=\frac{(l-2 a+d)(l-2 a+d-1)}{4(a+1)(a-d+1)} .
$$

Setting this equal to 1 , we find

$$
\begin{aligned}
a & =\frac{(l+d)^{2}}{4 l}+\frac{l-6 a+3 d-4}{4 l} \\
& =\frac{(l+d)^{2}}{4 l} \pm 4 \text { if }|d| \leqslant l \text { and } a \leqslant l .
\end{aligned}
$$

It follows that $R(l, a, d)=O\left(l^{\kappa} R\left(l,((r+1) / 2)^{2} l, r l\right)\right)$ if $d=a-b=r l$. By Stirling's formula, we now have

$$
\begin{aligned}
& R(l, a, a-b) \\
& =O\left(l ^ { \kappa } \operatorname { e x p } \left[l \left(-\left(\frac{1+r}{2}\right)^{2} \log \left(\frac{1+r}{2}\right)^{2}-\left(\frac{1-r}{2}\right)^{2} \log \left(\frac{1-r}{2}\right)^{2}\right.\right.\right. \\
& \left.\left.\left.-\left(\frac{1-r^{2}}{2}\right) \log \left(\frac{1-r^{2}}{2}\right)+\left(1-r^{2}\right) \frac{\log 2}{2}\right)\right]\right), \\
& =O\left(l^{k} \exp [l(2 \log 2-(1+r) \log (1+r)-(1-r) \log (1-r))]\right),
\end{aligned}
$$

as long as $r \neq \pm 1$, i.e. $d \neq \pm l$ (we treat these cases later).

Let $c=l / p=2 t(1+r t)^{-1}$. Then since $r t \leqslant 1 / 3, c \geqslant 2 t /(4 / 3) \geqslant 3 t / 2 \geqslant 0.45$. Hence by Lemma 2.6,

$$
\log \left(B_{p, l}\right)<n\left(\frac{1+r t}{2}\right)\left(X_{1} c^{2}+X_{2} c+x_{3}\right)+\kappa .
$$

Hence, by (2.3),

$$
\begin{aligned}
& \log \left(B_{p, l} R(l, a, a-b)\right) \\
& <\kappa \log n+n\left[\left(\frac{1+r t}{2}\right)\left(X_{1} c^{2}+X_{2} c+X_{3}\right)\right. \\
& \quad+t(2 \log 2-(1+r) \log (1+r)-(1-r) \log (1-r))] .
\end{aligned}
$$

Since $-1<r<1$ and $0.3 \leqslant t \leqslant 1$ and $|r t| \leqslant 1 / 3$, we now have by Lemma A.3 that

$$
\log \left(B_{p, l} R(l, a, a-b)\right)<1.3754 n+\kappa \log n .
$$

In case $d=l$ or $d=-l$, this still holds, because the numbers $B_{p, l}$ and $R(l, a, a-b)$ change by a factor of at most a polynomial in $n$ of bounded degree when $d$ is changed by \pm 1 and we already have (2.4) when $d=l-1$ and $d=-l+1$. From (2.4), the logarithm of the sum of (2.2) over all appropriate $l, a$ and $b(l \geqslant 0.3 M)$ is less than $1.3754 n+\kappa \log n$. Hence this is a bound on the logarithm of the number of different combinatorial types of $M$ in the case under consideration.

Now suppose that $t<0.3$. Let $C$ be a simple closed curve contained in the union of the invariants of $\alpha$ and intersecting each of them. Then by Lemma 2.4, $M_{2}(C)$ is 3-connected. Let $F^{\prime}$ be the face of $M_{2}(C)$ whose boundary is $C$, and suppose that $\alpha$ 
transfixes $a$ edges, flips $b$ edges and fixes $k$ faces of $M$. Then the edges of $M_{2}(C)$ incident with $F^{\prime}$ are the $a$ transfixed edges together with the $k$ intervals of $C$ contained in faces of $M$ fixed by $\alpha$. So $F^{\prime}$ has valency $a+k$. The edges of $M_{2}(C)$ are half of the $(n-a-b)$ edges of $M$ not fixed by $\alpha$, plus the $a$ transfixed edges, $b$ edges which are subintervals of the transfixed edges, and $k$ new edges in the fixed faces of $M$, making $(n+a+b+2 k) / 2$ in all. The number of combinatorially distinct possibilities for $M_{2}(C)$ with the face $F^{\prime}$ distinguished as special is at most the number of rooted 3-connected maps with the same number of edges which is $O\left(2^{n+a+b+2 k}\right)$ by Tutte's estimate (1.1).

Now if we are given $M_{2}(C)$ and are told which face is $F^{\prime}$, we still in general need to know precisely which vertices incident with $F^{\prime}$ are invariants of $\alpha$ and which edges incident with $F^{\prime}$ are transfixed by $\alpha$, before we can determine the combinatorial type of the original map $M$ (this extra information would certainly suffice, since it determines the $k$ edges lying in faces of $M$ and the $b$ vertices of $M_{2}(C)$ which are intersections of $C$ with edges of $M$ transfixed by $\alpha$ ). It is, however, enough to be told merely which $a$ edges incident with $F^{\prime}$ are transfixed by $\alpha$, for any invariant vertex not incident with such an edge has degree at least 4 in $M_{1}(C)$, whilst the vertices incident with $F^{\prime}$ which are not invariants of $\alpha$ are not vertices of $M$ and have degree 3. Thus, given $M_{2}(C)$ with $a$ and $F^{\prime}$ specified, the number of possibilities for $M$ is at most $\left(\begin{array}{c}a+k \\ a\end{array}\right)$ (recalling that $F^{\prime}$ has valency $a+k$ ) and $b$ is actually determined. Thus the number of possibilities for $M$ is

$$
O\left(2^{n+a+b+2 k}\left(\begin{array}{c}
a+k \\
k
\end{array}\right)\right)
$$

for given $a, b, k$ and $l$. Observe that each transfixed edge is incident with the face $F$ in $M_{1}$, and each invariant face is incident in $M$ with a noninvariant edge incident with $F$ in $M_{1}$. Hence $k+a \leqslant l$. Also, each edge of $M$ flipped by $\alpha$ is incident with two invariant faces, each of which is incident with at most one other flipped edge by Lemma 2.1 and so $b \leqslant k$. Hence, (2.5) is $O\left(2^{n+a+3 k}\left(\begin{array}{c}a+k \\ k\end{array}\right)\right)$ which is maximized over $a$ such that $k+a \leqslant l$ by putting $a+k=l$, i.e. it is

$$
O\left(2^{n+l+2 k}\left(\begin{array}{l}
l \\
k
\end{array}\right)\right)
$$

Now $2^{2 k}\left(\begin{array}{c}l \\ k\end{array}\right)$ is maximized when $k$ is approximately $4(l-k)$, i.e. $k=(4 / 5) l \pm 1$. So (2.5) is

$$
\begin{aligned}
O\left(l^{\kappa} 2^{n+(13 / 5) l}\left(\begin{array}{c}
l \\
l / 5
\end{array}\right)\right) \quad(\kappa \text { the ubiquitous constant }) \\
=O\left(l^{\kappa} 2^{n}\left(2^{13 / 5} \frac{1}{(1 / 5)^{1 / 5}(4 / 5)^{4 / 5}}\right)^{l}\right) \\
=O\left(n^{\kappa} 2^{n} 10^{t n}\right) \quad \text { recalling } l=t n \\
=O\left(n^{\kappa} 2^{n} 10^{.3 n}\right) \quad \text { as } t<0.3 .
\end{aligned}
$$


Summing over all appropriate $a, b, k, l$ only multiplies by $n^{\kappa}$ and consequently does not change the last bound. Thus the logarithm of the number of possibilities for $M$ in case $\alpha$ is plane-reflective and $t<0.3$, and hence for all $t$ in view of our treatment above of $l \geqslant 0.3$, is at most

$$
n(\log 2+0.3 \log 10)+\kappa \log n<1.38393 n .
$$

We next treat the case that $\alpha$ is antipodal. By Lemma 2.2, there is cell-cycle $S$, of faces and edges of $M$, which is fixed setwise by $\alpha$. Let $C$ be a simple closed curve intersecting each of the faces and edges in $S$ and no other cells of $M$, and intersecting each edge in $S$ precisely once. Then since each cell in $S$ is incident with just two others in $S, C$ intersects at most 2 edges incident with any one face. Since $\alpha$ has no invariants, $S$ contains at least 2 (and hence at least 3 ) faces. Thus, by Lemma 2.5, $N_{2}(C)$ is nonseparable and $N_{3}(C)$ is 3-connected. Since $\alpha$ has no invariants, it must permute the cells in $S$ in a cyclic fashion, and this permutation must correspond to a half-turn because $\alpha$ has order 2. As $\alpha$ is sense-preserving, it now follows that the cells of $M$ which are subsets if $R_{1}$ (the residual domain of $C$ which contans all points of $N_{2}(C)$ except for $U$ ) are mapped by $\alpha$ onto $R_{2}$, and vice-versa. Since every edge of $N_{2}(C)$ incident with $v$ corresponds to an edge in $S$ and since the edges in $S$ are permuted by $\alpha$ in a cyclic half-turn fashion by $\alpha$, it now follows that the combinatorial type of the map $N_{2}(C)$ determines the combinatorial type of the map $M$ if it is known which vertex of $N_{2}(C)$ is $v$. Hence the combinatorial type of the dual map $N_{2}(C)^{*}$ of $N_{2}(C)$ determines the combinatorial type of $M$, if it is known which face $F$ of $N_{2}(C)^{*}$ corresponds to $v$. For similar reasons, the combinatorial type of $N_{2}(C)$ determines the combinatorial type of $M$ if it is known which face, $F^{\prime}$ say, of $N_{2}(C)$ is $R_{2}$.

$F$ and $F^{\prime}$ have the same valency, say $l$. The number of edges in $N_{2}(C)$, and hence also $N_{2}(C)^{*}$, is $(1 / 2)(n-l)+l=(1 / 2)(n+l)$, and $N_{3}(C)$ has $l$ more edges, i.e. $(1 / 2)(n+3 l)$. Hence the number of combinatorial types of $M$ is at most the number of rooted 2-connected maps with $(1 / 2)(n+l)$ edges in which the face to the left of the root edge has valency $l$, and is also at most the number of rooted 3-connected planar maps with $(1 / 2)(n+3 l)$ edges, for the face to the left of the root edge can be specified to be $F$ or $F^{\prime}$ is required. Thus, the logarithm of the number of types of $M$ is at most

$$
(1 / 2)(n+l)\left(X_{1} c^{2}+X_{2} c+X_{3}\right)+\kappa
$$

by Lemma 2.6 if $c=2 l(n+l)^{-1}$ is at least 0.45 , and it is also bounded by

$$
(1 / 2)(n+3 l) \log 4+\kappa
$$

from (1.1).

Let $t=l / n$ and suppose firstly that $t \geqslant 0.3$. Then $c=2 t /(1+t) \geqslant .6 / 1.3>0.45$ and so (2.6) is valid for $t$ in this range. But now (2.6)

$$
\begin{aligned}
& n \frac{(t+1)}{2}\left(X_{1}\left(\frac{2 t}{1+t}\right)^{2}+X_{2}\left(\frac{2 t}{1+t}\right)+X_{3}\right)+\kappa \\
& \quad \leqslant n\left(X_{1} t^{2}+X_{2} t+(1 / 2)(t+1) X_{3}\right)+\kappa,
\end{aligned}
$$


(as $1+t \leqslant 2$ and $\left.X_{1}<0,2(1+t)^{-1} X_{1} \leqslant X_{1}\right)$. The minimum value of the parenthesized quadratic is

$$
\frac{-\left(X_{2}+\left(X_{3} / 2\right)\right)^{2}}{4 X_{1}}+X_{3} / 2=\frac{(.8676)^{2}}{3.916}+0.9656<1.16 \text {. }
$$

Thus (2.6) is less than $1.16 n+\kappa$.

Now suppose $t<0.3$. Then $(2.7)$ is less than $(0.95 \log 4) n+\kappa<1.3167 n+\kappa$. Multiplying by the $n$ possibilities for $l$ changes $\kappa$ only, and so the latter is a valid bound for the logarithm of the number of maps $M$ which possess an antipodal automorphism.

Finally suppose $\alpha$ is rotative. Then by Lemma 2.3 it has just two invariants, say $I_{1}$ and $I_{2}$. Let $S$ be a shortest cell-path in $M$ which begins at $I_{1}$ and ends at $I_{2}$ and contains no other vertices of $M$ than, perhaps, $I_{1}$ and $I_{2}$. Let $S^{\prime}=\alpha^{i}(S)$ be an image of $S$ under the $i$ th power of $\alpha$, where $i$ is less than the order of $\alpha$. Suppose $S$ and $S^{\prime}$ both contain the same cell, say $W$, other than $I_{1}$ and $I_{2}$. Then $\alpha^{i}(W)$ is in $S^{\prime}$. Since $\alpha^{i}$ is not the identity but is rotative, it fixes just $I_{1}$ and $i_{2}$ by Lemma 2.3 and hence $\alpha^{i}(W) \neq W$. But since $W$ has the same position in $S$ as $\alpha^{i}(W)$ has in $S^{\prime}$, this implies the existence of a shorter cell-path joining $I_{1}$ and $I_{2}$. Hence $S$ and $S^{\prime}$ are internally disjoint.

Let $i$ be chosen such that the number of cells adjacent to $I_{1}$ between the one in $S$ and the one in $S^{\prime}=\alpha^{i}(S)$ is minimized. Let $C^{-}$be a simple closed curve intersecting each of the cells in $S$ and $S^{\prime}$ and no other cells of $M$, and intersecting each edge in at most one point. Since $S$ and $S^{\prime}$ are internally-disjoint, no nonterminal cell in $S$ can even be incident with a nonterminal cell in $S^{\prime}$, for such an incidence would have to be a face-edge incidence, and the face involved would then have to be in both $S$ and $S^{\prime}$. Thus, $C^{-}$necessarily passes from $I_{1}$ along cells in $S$ to $I_{2}$, then along cells in $S^{\prime}$ back to $I_{1}$. Of the two residual domains of $C^{-}$(simply-connected open regions whose boundary is $C^{-}$), choose $R$ to be the one containing the minimum number of complete edges of $M$. Then by our choice of $i$ and of $R$, each power of $\alpha$ which is not the identity must map each cell of $M$ contained in $R$ outside of $R$; in fact, the part of $M$ within the closure of $R$, together with the order of $\alpha$, determine the combinatorial type of $M$ if the two cells $I_{1}$ and $I_{2}$, or the parts of them which are inside the closure of $R_{1}$ are known.

If $I_{1}$ and $I_{2}$ are both faces or edges, put $C=C^{-}$, and $C$ will then be a cell-cycle containing no vertices. But if $I_{1}$, say, is a vertex, we form $C$ from $C^{-}$by routing it around the faces and edges of $M$ within $R$ and incident with $I_{1}$ in order to eliminate $I_{1}$ from $C$ (we know $C^{-}$passes through only 2 faces incident with $I_{1}$ by the minimality of the length of $S$ ), and doing the same for $I_{2}$ if it is a vertex ( $I_{1}$ and $I_{2}$ cannot both be incident with the same face). Now $C$ is a cell-cycle containing no vertices of $M$. If we are given the structure of the part of $M$ in the residual domain $R^{\prime}$ of $C$ which lies within $R$, and we are told (i) the order of $\alpha$, (ii) which if any of $I_{1}$ and $I_{2}$ is a vertex, (iii) if $I_{1}$ or $I_{2}$ is a vertex, precisely which set of consecutive edges of $M$ in $R^{\prime}$ intersecting $C$ is the set of edges incident with it, and (iv) if $I_{1}$ or $I_{2}$ is a face or edge, precisely which face or edge intersecting $C$ it is, then we can reconstruct 
the structure of the closure of $R$, and hence the combinatorial type of $M$. The number of possibilities for the combination of (i), (ii), (iii) and (iv) combined is at most $n^{\kappa}$. Furthermore, if for the construction of $N_{2}(C)$ and $N_{3}(C)$ we choose $R_{1}$ to be $R^{\prime}$, then the combinatorial type of either $N_{2}(C)$ or $N_{3}(C)$ determines the structure of the part of $M$ within $R^{\prime}$ if the face of $N_{2}(C)$ containing $R_{2}$ and the vertex $v$ of $N_{3}(C)$ lying within $R_{2}$ are given. Hence, the number of distinct combinatorial types of $M$ is at most $n^{\kappa}$ times the number of distinct combinatorial possibilities for the dual map $N_{2}(C)^{*}$ of $N_{2}(C)$, and similar bound applies in terms of $N_{3}(C)$.

In case $C=C^{-}$, i.e. neither $I_{1}$ nor $I_{2}$ is a vertex, then $N_{2}(C)^{*}$ has $n / j+l / 2$ edges, where $j$ is the order of $\alpha$ and $l$ is the valency of the vertex $v$ of $N_{2}(C)$ and of the corresponding face of $N_{2}(C)^{*}$. This is at most $(1 / 2)(n+l)$. Also the number of edges in $N_{3}(C)$ is $(n / j)+3 l / 2 \leqslant(1 / 2)(n+3 l)$. It now follows as in the discussion of the antipodal case that the logarithm of the number of combinatorial types of $M$ in the case at hand exceed (2.6) by no more than $\kappa \log n$ if $2 l /(n+l)$ is at least 0.45 , and also exceed (2.7) by no more than $\kappa \log n$.

On the other hand, if $C \neq C^{-}$then the numbers of edges in $N_{2}(C)^{*}$ and $N_{3}(C)$ are strictly less than $(n / j)+(l / 2)$ and $(n / j)+(3 l / 2)$ respectively, and the conclusion of the preceding paragraph again applies. The argument given in the antipodal case now gives the bound $\exp (1.3167 n+\kappa)$ for the number of possibilities for $M$ in case $\alpha$ is rotative.

In conclusion we see that the number of 3-connected maps with $n$ edges and with a symmetry of minimal or prime order is at most $\exp (1.384 n)$ for $n$ sufficiently large. Each such map can be rooted in less than $4 n$ combinatorially distinct ways, whilst each of the maps with $n$ edges and no nontrival automorphism gives rise to $4 n$ distinct rooted maps. Thus

$$
4 n M(n)=R(n)+o(\exp (1.384 n))
$$

which proves Theorem 2.8 by Tutte's estimate (1.1) of $R_{n}$.

Appendix. In this appendix we establish a series of lemmas whose sole purpose is to give quadratic upper and lower bounds for $x \log x$ and related functions that arose in $\$ 2$.

Lemma A.1. For $0<x \leqslant 1$,

$$
x \log x \geqslant a_{1} x^{2}+b_{1} x+c_{1}
$$

for $.63 \leqslant x \leqslant 1$,

$$
x \log x \leqslant b_{2}(x-1)
$$

and for $1 \leqslant x \leqslant 2$,

$$
a_{3} x^{2}+b_{3} x+c_{3} \geqslant x \log x \geqslant a_{4} x^{2}+b_{4} x+c_{4},
$$

where

$$
\begin{array}{lll}
a_{1}=1.035 & b_{1}=-0.83 & c_{1}=-0.206 \\
1_{3}=0.333 & b_{2}=.78 & c_{3}=-0.71 \\
a_{4}=0.36 & b_{3}=0.387 & c_{4}=-0.67
\end{array}
$$


Proof. Let $f(x)=x \log x-a_{1} x^{2}-b_{1} x-c_{1}$. To show $f(x) \geqslant 0$, for $0 \leqslant x \leqslant 1$, we consider

$$
f^{\prime}(x)=1+\log x-2 a_{1} x-b_{1}, f^{\prime \prime}(x)=x^{-1}-2 a_{1} .
$$

Clearly $f^{\prime \prime}(x)<0$ for $x>\left(2 a_{1}\right)^{-1}$ and since $f(1)=.001$ and $f\left(\left(2 a_{1}\right)^{-1}\right)>0.01$ we have that $f(x)>0$ for $\left(2 a_{1}\right)^{-1} \leqslant x \leqslant 1$. Since $f^{\prime \prime}(x)>0$ for $0<x<\left(2 a_{1}\right)^{-1}$ it follows that $f^{\prime}(x)$ has at most one zero for $x$ in this range. Since $f^{\prime}(.296)<-0.00002$ and $f^{\prime}(.297)>0.00021$ the minimum of $f$ for $0<x<\left(2 a_{1}\right)^{-1}$ occurs for $.296<x<$ .297. For $x$ in this last range, however, both $x \log x$ and $a_{1} x^{2}+b_{1} x+c_{1}$ are decreasing. Hence in this range $x \log x>-0.360855$ and $a_{1} x^{2}+b_{1} x+c_{1}<-0.3609$ and so $f(x) \geqslant 0$ for $0 \leqslant x \leqslant 1$ and the first inequality is proved.

Let $f(x)=b_{2}(x-1)-x \log x$. Then $f^{\prime \prime}(x)=-x^{-1}<0$ for $.63 \leqslant x \leqslant 1$. Since $f(1)=0$ and $f(0.63)>.0005$ and since $f$ is convex we have the second inequality.

The proofs of the inequalities for $1 \leqslant x \leqslant 2$ are very similar to the proof of the first inequality. For the inequality involving $a_{3}, b_{3}$ and $c_{3}$ the corresponding $f(\geqslant 0)$ is convex for $1 \leqslant x \leqslant(.666)^{-1}$ and concave for $(.666)^{-1} \leqslant x \leqslant 2$. It must have one minimum between 1.817 and 1.818 and this minimum must be positive. For the inequality involving $a_{4}, b_{4}$ and $c_{4}$ the relevant $f$ has one minimum between 1.082 and 1.084 which is also positive.

The following function shall be important. Let

$$
\begin{aligned}
G(x, c)= & x \log x+(3-x-c) \log (3-x-c) \\
& -(1-x) \log (1-x)-2(x-c) \log (x-c) \\
& -(2 c-x) \log (2 c-x)-(2-c) \log (2-c) .
\end{aligned}
$$

LEMMA A.2. If $0.45 \leqslant c<x<1$ and $x<2 c$ then

$$
G(x, c)<X_{1} c^{2}+X_{2} c+X_{3}
$$

where $X_{1}=-0.979, X_{2}=-0.098, X_{3}=1.9312$.

Proof. First of all

$$
\exp \left(\frac{\partial G}{\partial x}\right)=\frac{x(1-x)(2 c-x)}{(3-x-c)(x-c)^{2}}
$$

We first show that

$$
x(1-x)(2 c-x)-(3-x-c)(x-c)^{2} \equiv h(x, c)
$$

is $\geqslant 0$ for $.45 \leqslant c<x \leqslant .63$, which will imply that $\partial G / \partial x \geqslant 0$ and so $G$ attains its maximum, for $c$ fixed, at some $x \geqslant .63$. Since

$$
\frac{\partial^{2} h}{\partial x^{2}}=12 x-6 c-8<0
$$

we certainly have that $h(x, c)$ attains its minimum value at $x=.63$ or $c$ for $.45 \leqslant c<x \leqslant .63$. Furthermore,

$$
\begin{gathered}
h(c, c)=c^{2}(1-c)>0, \\
h(x, c)=c^{3}+c^{2}(-x-3)+c\left(8 x-3 x^{2}\right)+2 x^{3}-4 x^{2} \\
h(.63, c)=c^{3}+c^{2}(-3.63)+c(3.8493)-1.087506
\end{gathered}
$$


and it remains to show that the minimum of $h(.63, c)$ on $.45 \leqslant c<.63$ is $\geqslant 0$. Note, however, that

$$
\frac{\partial^{2} h(.63, c)}{\partial c^{2}}=6 c-7.26<0
$$

and $h(.63, .45)=.000729$ and $h(.63, .63)=.146853$, thus $h(x, c) \geqslant 0$ for $.45 \leqslant c<x$ $\leqslant .63$, as we stated, and so $G$ attains its maximum value for $.45 \leqslant c<x<1$ at some $x \geqslant .63$.

In this case, however, $3-x-c<2$ and so by Lemma A.1, for fixed $c$,

$$
\begin{aligned}
G(x, c) \leqslant & b_{2}(x-1)+a_{3}(3-x-c)^{2}+b_{3}(3-x-c)+c_{3}-a_{1}(1-x)^{2} \\
& -2 a_{1}(x-c)^{2}-a_{1}(2 c-x)^{2}-a_{4}(2-c)^{2}-b_{1}-4 c_{1} \\
& -b 4(2-c)-c_{4}=a^{*} x^{2}+b^{*} x+c^{*} .
\end{aligned}
$$

The maximum value of this quadratic over all real $x$ is attained at $x=-b^{*} / 2 a^{*}$ and the max value is $c^{*}-\left(b^{*}\right)^{2} / 4 a^{*}$. Algebraic manipulation will express this maximum value as a quadratic in $c$ and it may be routinely verified that the coefficient of $c^{2}$ is less than $X_{1}$ and so on.

The next lemma, while only used once in the proof of Theorem 2.8 , is included here because it is similar to the previous two lemmas.

Lemma A.3. Let $0.3 \leqslant t \leqslant 1$ and $-1<r<1$ such that $|r t| \leqslant 1 / 3$. Put $c=$ $2 t(1+r t)^{-1}$. Then, with $X_{1}, X_{2}$ and $X_{3}$ defined as in Lemma A.2.

$$
\begin{aligned}
(1 / 2)(1+r t) & \left(X_{1} c^{2}+X_{2} c+X_{3}\right) \\
+t & {[2 \log 2-(1+r) \log (1+r)-(1-r) \log (1-r)]<1.3754 . }
\end{aligned}
$$

Proof. Let $f(r, t)$ denote the expression to be bounded. Since $X_{1}<0$ and $r t<1 / 3, X_{1}(1+r t)^{-1}<3 X_{1} / 4$ and so

$$
(1 / 2)(1+r t)\left(X_{1} c^{2}+X_{2} c+X_{3}\right)<(3 / 2) X_{1} t^{2}+X_{2} t+(1 / 2) X_{3}(1+r t) .
$$

Hence

$$
\begin{aligned}
f(r, t)< & t(2 \log 2-(1+r) \log (1+r)-(1-r) \log (1-r)) \\
& +(3 / 2) X_{1} t^{2}+X_{2} t+(1 / 2) X_{3}(1+r t) .
\end{aligned}
$$

Note that since $X_{3}>0,(1 / 2) X_{3}(1-r t)<(1 / 2) X_{3}(1+r t)$ for $r>0$, whilst the rest of this function is unchanged when $-r$ is substituted for $r$. So we know this function has its maximum for some $r>0$. Suppose $r>0$, then by Lemma A.1, with $1+r$ between 1 and $2,1-r$ between 0 and 1 we have $f(r, t)>Q(r, t)$ where $Q(r, t)$ is

$$
\begin{aligned}
& (3 / 2) X_{1} t^{2}+X_{2} t+(1 / 2) X_{3}(1+r t) \\
& +t\left[-a_{4}(1+r)^{2}-b_{4}(1+r)-c_{4}+2 \log 2-a_{1}(1-r)^{2}-b_{1}(1-r)-c_{1}\right] .
\end{aligned}
$$

Thus $Q(r, t)=t a^{*} r^{2}+t b^{*} r+c^{*}$ which attains its maximum value $c^{*}-t\left(b^{*}\right)^{2} / 4 a^{*}$. Now $-\left(b^{*}\right)^{2} / 4 a^{*}<0.252$ and so (recall $t>0$ )

$$
f(r, t)<0.252 t+c^{*}=R_{1} t^{2}+R_{2} t+R_{3}
$$

where $R_{1}=-1.4685, R_{2} \leqslant 1.5514$ and $R_{3}=0.9656$. The maximum value of this quadratic is $<1.3754$ as required. 


\section{REFERENCES}

1. W. G. Brown, Enumeration of non-separable planar maps, Canad. J. Math. 15 (1963), 526-545.

2. P. J. Frederico, The number of polyhedra, Philips Res. Rep. 30 (1975), 220-231.

3. B. Grünbaum, Convex polytopes, Interscience [Wiley], New York, 1967.

4. V. A. Liskovets, Enumeration of nonisomorphic planar maps, J. Graph Theory 5 (1981), 115-117.

5. P. Mani, Automorphismen von polyedrischen Graphen, Math. Ann. 192 (1971), 279-303.

6. W. T. Tutte, The enumerative theory of planar maps, A Survey of Conbinatorial Theory (J. N. Srivastava et al., editors), North-Holland, Amsterdam, 1973, pp. 437-448.

7. __ A census of planar maps, Canad. J. Math. 15 (1963), 249-271.

8. On the enumeration of convex polyhedra, J. Combin. Theory 28 (1980), 105-126.

9. N. C. Wormald, Counting unrooted planar maps, Discrete Math. 36 (1981), 117-137.

10. ${ }_{1}$ On the number of planar maps, Canad. J. Math. 33 (1981), 1-7.

DEPARTMENT OF COMBINATORICS AND OPTIMISATION, UNIVERSITY OF WATERLOO, WATERLOO, ONTARIO, CANADA N2L 3G1

Department of Mathematics, University of Newcastle, Newcastle, Australia 